J. Dairy Sci. 96:5723-5733

http://dx.doi.org/10.3168/jds.2012-6460

(c) American Dairy Science Association ${ }^{\circledR}, 2013$.

\title{
Implementation of multivariate cumulative sum control charts in mastitis and lameness monitoring
}

\author{
Bettina Miekley, ${ }^{* 1}$ Eckhard Stamer, $\dagger$ Imke Traulsen, ${ }^{*}$ and Joachim Krieter* \\ *Institute of Animal Breeding and Husbandry, Hermann-Rodewald-Straße 6, D-24118 Kiel, Germany \\ †TiDa Tier und Daten GmbH, D-24259 Westensee/Brux, Germany
}

\begin{abstract}
This study analyzed the methodology and applicability of multivariate cumulative sum (MCUSUM) charts for early mastitis and lameness detection. Data used were recorded on the Karkendamm dairy research farm, Germany, between August 2008 and December 2010. Data of 328 and 315 cows in their first $200 \mathrm{~d}$ in milk were analyzed for mastitis and lameness detection, respectively. Mastitis as well as lameness was specified according to veterinary treatments. Both diseases were defined as disease blocks. Different disease definitions for mastitis and lameness ( 2 for mastitis and 3 for lameness) varied solely in the sequence length of the blocks. Only the days before the treatment were included in the disease blocks. Milk electrical conductivity, milk yield, and feeding patterns (feed intake, number of trough visits, and feeding time) were used for the recognition of mastitis. Pedometer activity and feeding patterns were used for lameness detection. To exclude biological trends and obtain independent observations, the values of each input variable were either preprocessed by wavelet filters or a multivariate vector autoregressive model. The residuals generated between the observed and filtered or observed and forecast values, respectively, were then transferred to a classic or self-starting MCUSUM chart. The combination of the 2 preprocessing methods with each of the 2 MCUSUM sum charts resulted in 4 combined monitoring systems. For mastitis as well as lameness detection requiring a block sensitivity of at least $70 \%$, all 4 of the combined monitoring systems used revealed similar results within each of the disease definitions. Specificities of 73 to $80 \%$ and error rates of $99.6 \%$ were achieved for mastitis. The results for lameness showed that the definitions used obtained specificities of up to $81 \%$ and error rates of $99.1 \%$. The results indicate that the monitoring systems with these study characteristics have appealing features for masti-
\end{abstract}

Received December 7, 2012

Accepted June 3, 2013.

${ }^{1}$ Corresponding author: bmiekley@tierzucht.uni-kiel.de tis and lameness detection. However, they are not yet directly applicable for practical implementations.

Key words: disease detection, multivariate cumulative sum chart, wavelet, vector autoregression

\section{INTRODUCTION}

In dairy production, the stockman's health management aims for the early detection and intervention of diseases, such as mastitis and lameness. Mastitis and lameness still remain the most frequent and costly diseases in the dairy industry in terms of economics and animal welfare (Kramer et al., 2009). The early detection of and intervention against mastitis and lameness reduces veterinary fees, losses in milk yield (MY) and milk quality, and increases the cure rate of the infected animals (Milner et al., 1997).

However, with growing herd size, the classic detection method of visual observations has become more difficult and time consuming. Thus, a need exists to support the farmer's observations by applying improved and automated detection of diseases (de Mol et al., 1997). Automated detection is possible using sensor measurements and information from a management information system.

In industrial and chemical production, the concepts of statistical process control and, more specifically, control charts have already been used as one of the major statistical process control tools (De Vries and Reneau, 2010; Mertens et al., 2011). The availability of regularly recorded data on the variables of dairy production processes creates the opportunity to develop such control charts to support the stockman in his daily management tasks (Mertens et al., 2011). Although the concepts of control charts have been proposed for use in agricultural science since the 1970s (De Vries and Reneau, 2010), they have not been widely applied in this field. Recently, the use of control charts in livestock production has been regaining interest (Mertens et al., 2011). Lukas et al. (2009) and Miekley et al. (2012), for example, used self-starting cumulative sum (CUSUM) charts for mastitis detection, whereas de Vries and Conlin (2003) used a Shewhart and a classic CUSUM 
chart for estrus detection. However, the charts of these studies are univariate control charts.

In manufacturing industries as well as agricultural science, several quality variables may be controlled simultaneously with one chart, taking any correlation between the variables into account (Hawkins and Maboudou-Tchao, 2007; Waterhouse et al., 2010). Therefore, in industrial process control, multivariate quality control charts have been established. Such multivariate methods use the relationship between the components of a multivariate process to generate more powerful algorithms compared with univariate approaches. The aim is to detect changes in the process that can hardly be detected by a univariate attempt (Bodnar and Schmid, 2007)

Numerous multivariate charts and their extensions are presently available. These charts can be grouped into 3 broad categories, namely Hotelling's $\mathrm{T}^{2}$, multivariate exponentially weighted moving average, and multivariate CUSUM (MCUSUM) charts. Multivariate CUSUM charts have proved particularly powerful (Patel and Divecha, 2010). However, control charts in general are sensitive to trends and autocorrelation within data (Montgomery, 2009). Thus, preprocessing methods (e.g., filters or time series models) play an important role in process control using MCUSUM charts.

Therefore, this paper aims to provide an introduction to the concepts and possible preprocessing methods of MSUSUM charts as well as their applicability in the early detection of mastitis and lameness based on practically recorded farm data. For preprocessing, wavelet filters and multivariate vector autoregressive models were chosen for mastitis and lameness detection, respectively. Besides the classical MCUSUM chart, a selfstarting approach of the MCUSUM will be discussed.

\section{MATERIALS AND METHODS}

\section{Data}

Data used was recorded on the Karkendamm dairy research farm, located in Northern Germany, between August 2008 and December 2010. About 66,000 cowdays from 338 and 315 cows in their first 200 DIM were analyzed for mastitis and lameness detection, respec- tively. Milk parameters [such as MY and milk electrical conductivity (MEC)] have been used for mastitis detection in recent studies (Cavero et al., 2008; Lukas et al., 2009), whereas the activity of cows has been used for lameness detection (Kramer et al., 2009). Recently, feed intake and its corresponding behavior have been reported to be linked to a cow's health status (González et al., 2008; Lukas et al., 2008). Therefore, MEC, MY, and feeding patterns (feed intake, number of trough visits, and feeding time) were used for the recognition of mastitis. Pedometer activity and feeding patterns were used for lameness detection.

Milking took place in a rotary milking parlor manufactured by GEA Farm Technologies GmbH (Bönen, Germany). Cows were milked twice daily. Milk yield and MEC were measured using the Metatron P21 milk meter (GEA Farm Technologies GmbH) at every milking. Activity was measured using pedometers (GEA Farm Technologies $\mathrm{GmbH}$ ), which recorded activity in 2-h periods. Cows were fed in individual feeding troughs. The feeding trough was developed and installed by the Institute of Animal Breeding and Husbandry, University of Kiel (Kiel, Germany). Each visit to the feeding troughs was recorded and the amounts of consumed feed were accumulated to daily intakes. Extreme values (mainly for the trait feed intake) that deviated by more than \pm 4 standard deviations were excluded from the data set. To adjust for different timescales between the indicator variables, daily MY, weighted MEC per day, and average daily activity rates were calculated (Table 1). Furthermore, high pedometer activity due to documented and progesterone-measured estrus events was excluded from the data set.

Medical treatments of diseases were documented constantly by veterinarians and farm staff. Different categories for mastitis (e.g., Staphylococcus aureus or Escherichia coli mastitis) and for claw and leg diseases (e. g. digital dermatitis or sole ulcer) were identified. Due to the low frequencies of diseased cows within these categories, the categories were combined to form cases of mastitis and lameness, respectively. These cases were defined as the target characteristic to be distinguished from the healthy observation in the data. In the case of mastitis, 238 cows without any mastitis treatment during their first 200 DIM as well as 100 treated cows were

Table 1. Descriptive statistics of the traits showing the number of observations (n), mean, SD, and minimum (Min) and maximum (Max) values

\begin{tabular}{|c|c|c|c|c|c|}
\hline Item & $\mathrm{n}$ & Mean & SD & Min & Max \\
\hline Milk electrical conductivity (reference values) & 63,703 & 496.2 & 34.2 & 320.0 & 874.0 \\
\hline Activity (contacts/d) & 58,898 & 30.7 & 10.0 & 4.5 & 70.0 \\
\hline Time at trough $(\min / \mathrm{d})$ & 48,642 & 171.2 & 58.5 & 13.0 & 295.0 \\
\hline Trough visits (no./d) & 48,647 & 44.5 & 15.6 & 5.2 & 117.0 \\
\hline
\end{tabular}


Table 2. Number of evaluated days of health and days of disease (with percentages in parentheses) as well as the average number of ill and healthy cows per day according to the different mastitis/lameness definitions considered

\begin{tabular}{lcccc}
\hline Item & \multicolumn{1}{c}{$\begin{array}{c}\text { Days } \\
\text { of health }\end{array}$} & $\begin{array}{c}\text { Days } \\
\text { of disease }\end{array}$ & $\begin{array}{c}\text { Ill cows } \\
(\text { no./d) }\end{array}$ & $\begin{array}{c}\text { Healthy } \\
\text { cows (no./d) }\end{array}$ \\
\hline $\begin{array}{l}\text { Mastitis } \\
\text { Mastitis }+3\end{array}$ & $48,138(99.7)$ & $158(0.3)$ & 0.4 & 78.6 \\
Mastitis +4 & $48,090(99.6)$ & $209(0.4)$ & 0.6 & 78.4 \\
Lameness & & & & \\
Lame +3 & $39,642(99.2)$ & $340(0.8)$ & 0.5 & 67.6 \\
Lame +5 & $39,452(98.7)$ & $519(1.3)$ & 0.7 & 67.4 \\
Lame +7 & $39,272(98.3)$ & $696(1.7)$ & 1.0 & 67.1 \\
\hline
\end{tabular}

${ }^{1}$ Mastitis $+3=$ day of mastitis treatment plus $3 \mathrm{~d}$ before the treatment; mastitis $+4=$ day of mastitis treatment plus $4 \mathrm{~d}$ before the treatment; lame $+3=$ day of lameness treatment plus $3 \mathrm{~d}$ before the treatment; lame $+5=$ day of lameness treatment plus $5 \mathrm{~d}$ before the treatment; lame $+7=$ day of lameness treatment plus $7 \mathrm{~d}$ before the treatment.

analyzed. The number of treated cows due to lameness was 142 .

\section{Disease Definitions}

Diseases were defined as disease blocks (i.e., an uninterrupted sequence of "days of disease"; Cavero et al., 2008; Kramer et al., 2009). The treatments served as a basis for these disease blocks and the different definitions varied solely by the sequence length of the blocks. As the focus of this study was on early disease detection, only the days before a treatment were included in a disease block (Kramer et al., 2009). If at least one alarm was generated by the monitoring system within the block, it was considered as detected.

Mastitis Definition. Cows were selected for veterinary treatment by the farm staff based on observable signs of mastitis infection. Two variants of mastitis definition were used in this study:

- Mastitis + 3: day of mastitis treatment plus $3 \mathrm{~d}$ before the treatment;

- Mastitis + 4: day of mastitis treatment plus $4 \mathrm{~d}$ before the treatment.

The days in the data set were classified as "days of health" or "days of disease" according to Cavero et al. (2007; (Table 2). The day of treatment as well as 3 or $4 \mathrm{~d}$ before were defined as days of disease. To give consideration to the withdrawal period without any observation, at least $7 \mathrm{~d}$ after the last treatment of a mastitis case were not used for the analysis. After this period, cows were considered to be healthy. Combining treatment and 3 or $4 \mathrm{~d}$ before resulted in 115 mastitis blocks. Besides the number of days of health and days of disease, Table 2 a shows the average number of ill and healthy cows per day for both mastitis definitions.

Lameness Definition. For veterinary treatment, lame cows were also selected by the farm staff based on observable signs. Lameness was defined using disease blocks analogous to the mastitis definitions. The different definitions varied in the length of the disease blocks:

- Lame + 3: day of lameness treatment plus $3 \mathrm{~d}$ before the treatment;

- Lame + 5: day of lameness treatment plus $5 \mathrm{~d}$ before the treatment;

- Lame + 7: day of lameness treatment plus $7 \mathrm{~d}$ before the treatment.

All medicated cows were again observed by a veterinarian $1 \mathrm{wk}$ after treatment. Thus, all days between treatment and a follow-up examination were set to days of disease. If the follow-up examination proved negative, cows were considered healthy. Otherwise, the period of days of disease after the first treatment was lengthened for another week. Thus, 210 disease blocks were observed. For all lameness definitions, the number of days of health and days of disease as well as the number of lame and healthy cows per day are shown in Table 2.

\section{Methods}

Wavelet Filters and Multivariate Vector Autoregressive Models. Statistical process control traditionally assumes that process measurements over time are stationary (without trend) and independent (Montgomery, 2009). Typically, biological data are mostly nonstationary and autocorrelated (Montgomery, 2009; Mertens et al., 2011). In this case, the common approach is to reduce or remove the trend and the autocorrelation from the process by using appropriate time series models or a model-free approach (e.g., filters) and to use the residuals to monitor the process (Montgomery, 2009). 
Because the residuals would be stationary and uncorrelated, the assumption of traditional control charts would be satisfied. According to Bodnar and Schmid (2007), multivariate control charts based on residuals simplify the correlation structure and thus the determination of the control design.

A relatively easy implementable and univariate way of generating residuals using a model-free approach is described by Miekley et al. (2012) using wavelet filters. In short, wavelet filtering identifies trends and noise in the input variable, and then reconstructs this time series without those components. All calculations were computed using the MATLAB Wavelet Toolbox (MathWorks Inc., 2010). For more detailed information on wavelet analysis, refer to Daubechies (1990), Gençay et al. (2002), and Miekley et al. (2012).

In the present study, the input variables of each cow were individually filtered using Daubechies fourthorder wavelet for the first filtering step. Residuals (differences between the observed and filtered values) were calculated and then used for individual monitoring of the cow based on multivariate cumulative sum charts.

In the case of univariate preprocessing of data (e.g., wavelets), possible dependencies between the input variables are not considered. In multivariate cases, the commonly used multivariate time series model is the vector autoregressive (VAR) model (Lütkepohl, 2007). The VAR model is the extension of the univariate autoregressive model to multivariate data, describing relationships between several time series variables. In this model, each variable not only depends on its past value, but also on the past value of other variables taking the calculated autoregressive parameters into account (Wutsqa et al., 2006; Athanasopoulos and Vahid, 2008). Vector autoregressive models themselves assume that the input data are stationary (mean and variance do not change over time, trend; Lütkepohl, 1991; Athanasopoulos and Vahid, 2008). Following Makridakis et al. (1988), each input variable was differenced once at cow level to achieve stationarity. Further differencing was not necessary due to over differencing (data becomes nonstationary again). Then, the aim of VAR models is to approximate the actual process resulting in forecast values. These forecast values can be used to generate more reliable but also independent residuals (differences between the observed and forecast value). The multivariate VAR model was also applied to individual cows in this study. The resulting residuals were used for mastitis and lameness detection for each cow. For more detailed information on multivariate VAR models, refer to Pfaff (2008).

A graphical summary of each step of the monitoring system using wavelet filtering or multivariate VAR

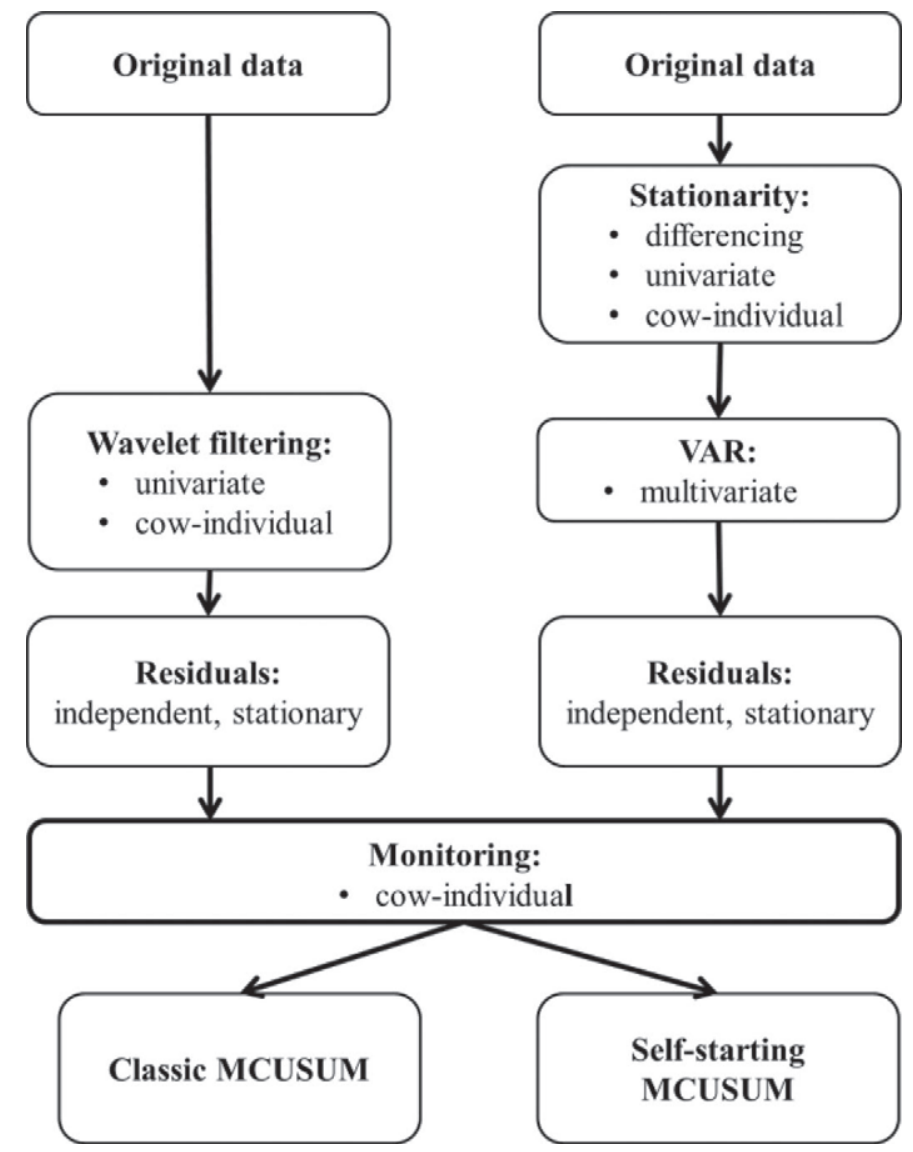

Figure 1. General procedure of the detection system using either wavelet filtering or vector autoregressive (VAR) models for mastitis or lameness detection with multivariate cumulative sum (MCUSUM) charts.

models combined with MCUSUM charts is shown in Figure 1. Each succeeding step is based on individual cow analysis.

MCUSUM. Two different MCUSUM charts were used to monitor the residuals: (1) the classic MCUSUM and (2) the self-starting MCUSUM chart. Both charts are presented in this section.

Taking the relationship between the indicator variables into account, the aim of the MCUSUM chart in general is to plot multivariate observations over time with one chart to see whether a process is in control (i.e., a cow is healthy).

The multivariate control chart of Pignatiello and Runger (1990), in this study also referred to as classic MCUSUM, is widely used in practical applications due to its good ability to detect shifts of small and medium size in a process of interest (da Cunha Alves et al., 2010). The one-sided multivariate CUSUM chart (MC2; Figure 2) of Pignatiello and Runger (1990) is constructed by 


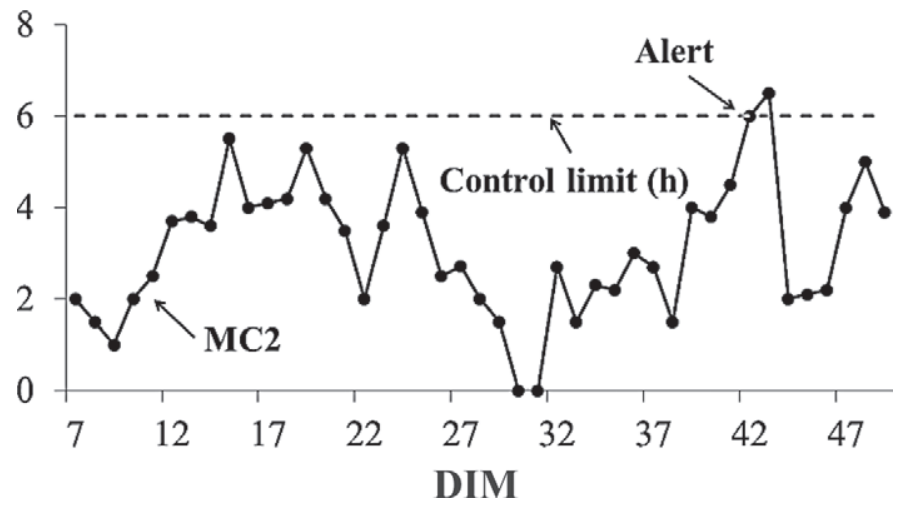

Figure 2. Example of the multivariate cumulative sum chart (MC2) of Pignatiello and Runger (1990) based on one cow.

$$
\mathrm{MC} 2_{\mathrm{t}}=\max \left(0, \mathrm{MC} 2_{\mathrm{t}-1}+\mathrm{D}_{\mathrm{t}}^{2}-\mathrm{k}\right), \text { with } \mathrm{MC} 2_{0}=0 .
$$

$\mathrm{D}_{\mathrm{t}}^{2}$ is calculated as follows:

$$
\mathrm{D}_{\mathrm{t}}^{2}=\left(\mathrm{X}_{\mathrm{t}}-\mu\right)^{\prime} \boldsymbol{\Sigma}^{-1}\left(\mathrm{X}_{\mathrm{t}}-\mu\right)
$$

where $\mathrm{X}_{\mathrm{t}}$ denotes the $\mathbf{p}^{*} 1$ vector of the residuals from the input variables (e.g., mastitis: MY, MEC, and 3 feeding patterns; $\mathbf{p}=5$ ) at time t for a single cow. The MCUSUM assumes that the input variables of $\mathrm{X}_{\mathrm{t}}$ are independent and identically distributed multivariate normal random vectors (Pignatiello and Runger, 1990). The independency is ensured through the use of residuals generated by the methods of the aforementioned section. The residuals were also checked for multivariate normality, which was met by both data sets. The inverse of the covariance matrix is represented by $\boldsymbol{\Sigma}^{-1}$, whereas $\mu$ is the mean of each residual input variable from each cow while the animal is considered to be healthy. To establish $\boldsymbol{\Sigma}^{-1}$ and $\mu$ based on healthy observations, prior information (Waterhouse et al., 2010) of each cow is needed. Due to the lack of historical data on each cow, both parameters were calculated based on the given data sets. The constant $\mathrm{k}$ is called the reference value, which is related to the magnitude of change to be detected (Pignatiello and Runger, 1990; da Cunha Alves et al., 2010). In the present study, $\mathrm{k}$ was tested for the values of 0.2 to 3.2 and then set to 1.2 for mastitis and lameness detection. To identify when the mean has shifted from the specified values, a control limit is plotted on the charts (Figure 2). It is determined by the $h$ value, also called the decision interval. The value of $h$ was varied from value 0.1 to 18 .

To run the MCUSUM without any preassumptions about the mean of each cow, a self-starting transformation based on the univariate self-starting CUSUM
(Montgomery, 2009) was also applied in this analysis. Self-starting methods update the parameter estimates (mean and variation) with each new observation of each input variable and cow. Both are updated by calculating a running mean and running variation (Hawkins and Olwell, 1998; Montgomery, 2009) at cow level. The standardization of each observation, using the running mean and standard deviation of the preceding observations, gives a standardized variate of each input variable at time t. These variates are then transformed to become independent and follow the standard normal distribution (Montgomery, 2009). Therefore, the calculation of $\mathrm{D}_{\mathrm{t}}^{2}$ changes to

$$
\mathrm{D}_{\mathrm{t}}^{2}=\mathrm{D}_{\mathrm{t}}^{\prime} \boldsymbol{\Sigma}^{-1} \mathrm{D}_{\mathrm{t}}
$$

where $\mathrm{D}_{t}$ stands for the transformed variates of the residual of each input variable and cow at time t. For calculation of MC2 at time $t, \mathrm{D}_{\mathrm{t}}^{2}$ was then handled as any $\mathrm{D}_{\mathrm{t}}^{2}$ for a classic MCUSUM chart and was applied and plotted according to the aforementioned formula. The covariance matrix $(\boldsymbol{\Sigma})$ was the only parameter assumed to be known beforehand. It was calculated in the same way as the classic MCUSUM but this time based on the transformed residuals. The values for the reference value (k) as well as the decision interval (h) were varied in the same manner as for the classic MCUSUM.

For further information on self-starting transformation for univariate CUSUM charts, refer to Hawkins and Olwell (1998) or Montgomery (2009). To exclude high (detected) values from further calculations, both MCUSUM charts were reset after each $\mathrm{MC} 2$ value above the upper control limit (Montgomery, 2009). All of the VAR and MCUSUM calculations were computed using SAS software (SAS Institute, 2009).

\section{Test Procedure}

The MCUSUM chart provided an alert whenever values above the control limit of the chart occurred (Figure 2). System performance was assessed by comparing these alerts with the actual occurrence of disease.

The corresponding day of observation was classified as true positive (TP) if the limit was exceeded on a day of disease, whereas an undetected day of disease was classified as false negative $(\mathbf{F N})$. Each day in a healthy period was considered as a true negative (TN) case if no alert was generated and as a false positive (FP) case if an alert was given. The accuracy of these procedures was evaluated by the parameters sensitivity, block sensitivity, specificity, and error rate. Sensitivity represents the percentage of correctly detected days of disease of all days of disease: 


$$
\text { sensitivity }=\frac{\mathrm{TP}}{\mathrm{TP}+\mathrm{FN}} \times 100 \text {. }
$$

For disease detection, it was not important for all days of a disease block to be recognized, but it was crucial for mastitis or lameness to be detected at all and early on. Therefore, the block sensitivity was deemed considerably more important than sensitivity. For the block sensitivity, each disease block was considered a TP case if one or more alerts were given within the defined disease block and an FN case otherwise (Cavero et al., 2007; Kramer et al., 2009). The specificity indicates the percentage of correctly found days of health from all the days of health:

$$
\text { specificity }=\frac{\mathrm{TN}}{\mathrm{TN}+\mathrm{FP}} \times 100
$$

The error rate represents the percentage of days outside the disease periods from all the days where an alarm was produced:

$$
\text { error rate }=\frac{\mathrm{FP}}{\mathrm{FP}+\mathrm{TP}} \times 100
$$

In addition, the number of TP as well as FP cows per day is given. The TP and FP cows per day signify the average number of rightly and wrongly diseasedregistered cows per day, respectively.

\section{RESULTS}

Working with varying control limits, as in this study, each value generates its own block sensitivity, specific- ity and error rate. According to Hogeveen et al. (2010), the sensitivity of an automatic milking system should be at least $80 \%$, whereas for milking parlors in which the herdsmen are still present, as in Karkendamm, the sensitivity may be lower. Thus, the block sensitivity for mastitis and lameness detection was chosen to be at least 70\%, which is in concordance with Kramer et al. (2009).

Table 3 shows the results for the combined monitoring system wavelet filters and MCUSUM achieving at least $70 \%$ block sensitivity. For the first mastitis definition (mastitis +3 ), the specificity was $71.4 \%$ with an error rate of $99.6 \%$. The amount of FP cows per day was 19.5 animals at an average herd size of around 79.7 cows per day.

Compared with mastitis +3 , the second mastitis definition (mastitis +4 ) showed better results. At a minimum of $70 \%$ block sensitivity $(70.2 \%)$ the specificity was higher $(78.9 \%)$ and the number of FP cows per day (14.4 cows) was lower than the first mastitis definition used.

Lameness detection with wavelets and MCUSUM charts (Table $3 \mathrm{~b}$ ) showed more clearly than the mastitis definitions that the longer the disease period under consideration was, the better the monitoring system performed. Lame +3 , for example, revealed the lowest results of all of the lameness definitions. On the other hand, lame +7 performed best, having the highest specificity value, the lowest error rate, and lowest number of cows per day falsely detected as ill (10.9 FP cows per day) at an average herd size of 68 cows per day. Table 4 displays the results for mastitis and lameness detection using the residuals of the multivariate VAR model combined with MCUSUM charts, reaching at least $70 \%$ block sensitivity.

Table 3. Results of the wavelet filtering combined with classic multivariate cumulative sum (MCUSUM) charts requiring at least $70 \%$ block sensitivity

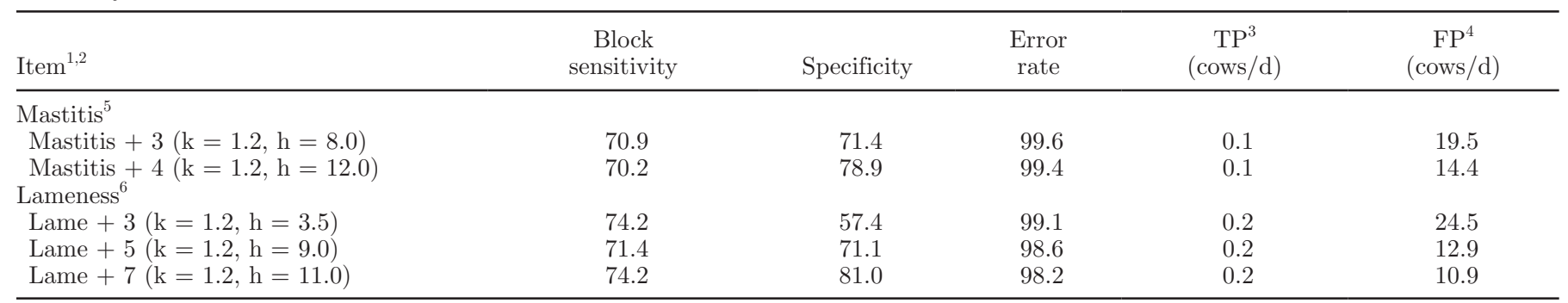

\footnotetext{
${ }^{1}$ Mastitis $+3=$ day of mastitis treatment plus $3 \mathrm{~d}$ before the treatment; mastitis $+4=$ day of mastitis treatment plus $4 \mathrm{~d}$ before the treatment; lame $+3=$ day of lameness treatment plus $3 \mathrm{~d}$ before the treatment; lame $+5=$ day of lameness treatment plus $5 \mathrm{~d}$ before the treatment; lame $+7=$ day of lameness treatment plus $7 \mathrm{~d}$ before the treatment.

${ }^{2} \mathrm{k}=$ reference value constant, which is related to the magnitude of change to be detected; $\mathrm{h}=$ decision interval, which varied from a value of 0.1 to 18 .

${ }^{3}$ True positive.

${ }^{4}$ False positive.

${ }^{5}$ Average herd size: 79.7 cows per day.

${ }^{6}$ Average herd size: 68.1 cows per day.
} 
Table 4. Results of the multivariate vector autoregressive (VAR) models combined with classic multivariate cumulative sum (MCUSUM) charts requiring at least $70 \%$ block sensitivity

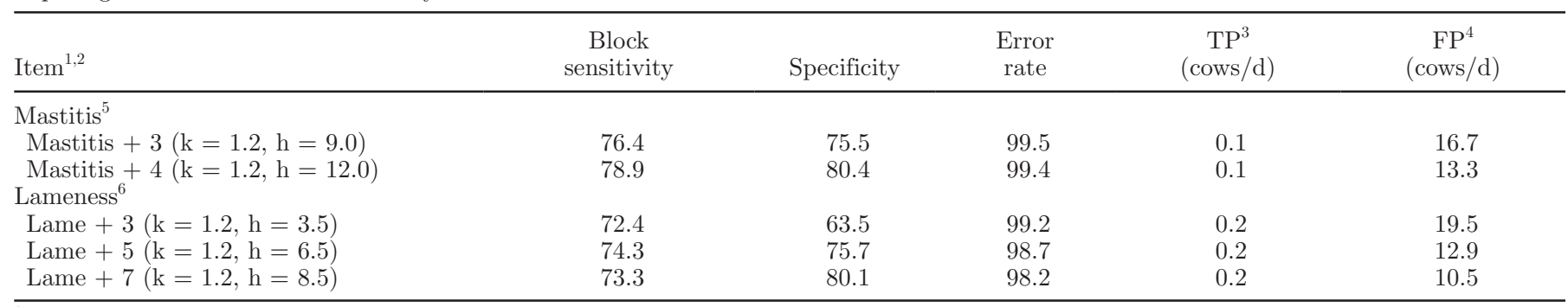

${ }^{1}$ Mastitis $+3=$ day of mastitis treatment plus $3 \mathrm{~d}$ before the treatment; mastitis $+4=$ day of mastitis treatment plus $4 \mathrm{~d}$ before the treatment; lame $+3=$ day of lameness treatment plus $3 \mathrm{~d}$ before the treatment; lame $+5=$ day of lameness treatment plus $5 \mathrm{~d}$ before the treatment; lame $+7=$ day of lameness treatment plus $7 \mathrm{~d}$ before the treatment.

${ }^{2} \mathrm{k}=$ reference value constant, which is related to the magnitude of change to be detected; $\mathrm{h}=$ decision interval, which varied from a value of 0.1 to 18 .

${ }^{3}$ True positive.

${ }^{4}$ False positive.

${ }^{5}$ Average herd size: 78.6 cows per day.

${ }^{6}$ Average herd size: 67.3 cows per day.

The analysis of mastitis detection showed that mastitis +3 performed more poorly compared with mastitis +4 . The specificity (e.g., of mastitis +3 ) was $75.5 \%$ with $16.7 \mathrm{FP}$ cows per day, whereas the specificity and number of FP cows per day of mastitis +4 was $80.4 \%$ and 13.3, respectively. Considering the specificities and number of FP cows per day, both mastitis definitions analyzed by this combined monitoring system revealed slightly better system performance than mastitis +3 and mastitis +4 monitored by wavelets and MCUSUM charts (Table 3). Lameness results based on the VAR model (Table 4) combined with MCUSUM charts show that lame +5 and lame +7 differ only marginally from the results of the wavelet and MCUSUM monitoring system.

The results of the monitoring system based on wavelet filtering in combination with a self-starting MCUSUM (Table 5) reveal that, for both diseases within their definitions, specificities, error rates as well as the number of FP cows per day did not deviate much from the results of wavelets and classic MCUSUM. Mastitis +4 of the self-starting approach, for example, reached a specificity of $77.4 \%$ with an error rate of $99.5 \%$ and 15.2 FP cows per day, whereas the same definition using wavelets with a classic MCUSUM attained a specificity of $78.9 \%$, an error rate of $99.4 \%$ and $14.4 \mathrm{FP}$ cows per day.

Examining the results of the VAR models combined with a self-starting MCUSUM (Table 6) for both diseases shows again that the values achieved were close to the outcome of the VAR and classic MCUSUM chart of each definition used. The findings of lame +5 at the self-starting analysis, for example, almost equals the specificity, error rate, and the number of FP cows per day of the monitoring system of VAR and MCUSUM charts.

\section{DISCUSSION}

The minimum requirement for the monitoring systems analyzed in this study is a block sensitivity of at least 70\% (Kramer et al., 2009; Miekley et al., 2012). For both diseases and for all of the 4 detection systems used (wavelet filtering or the VAR model combined with a classic MCUSUM or self-starting transformed MCUSUM), block sensitivities were above this system requirement. Thus, the monitoring systems provided sufficient detection performance for mastitis as well as for lameness. Specificities, however, were only around $80 \%$ and below. Therefore, the error rates were too high at about $99 \%$. Affecting error rate and specificity, the amount of FP alerts was also high in the present study. The higher the number of FP cows per day, the greater workload the herdsman has, leading to a loss of confidence in the monitoring system. Such unfavorable results obtained by the monitoring systems analyzed in this study can be caused by several reasons.

An important factor that influences classification results is the disease definition. Again, an animal was considered to be ill if a treatment occurred. These treatments were carried out by a qualified veterinarian and can, therefore, be considered reliable. However, cows showing no obvious, visible signs of disease and thus overlooked by the herdsmen do exist (Højsgaard and Friggens, 2010; Kamphuis et al., 2010). Although such cows are ill, they might be classified as FP cows. To avoid ill but untreated mastitic cows, Miekley et al. (2012) and Kramer et al. (2009) combined treatments 
Table 5. Results of the wavelet filtering combined with self-starting multivariate cumulative sum (MCUSUM) charts requiring at least $70 \%$ block sensitivity

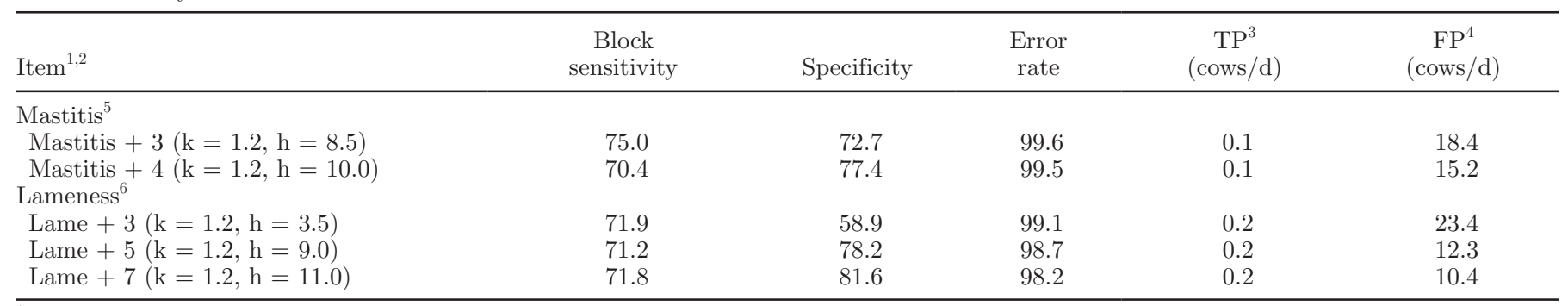

${ }^{1}$ Mastitis $+3=$ day of mastitis treatment plus $3 \mathrm{~d}$ before the treatment; mastitis $+4=$ day of mastitis treatment plus $4 \mathrm{~d}$ before the treatment; lame $+3=$ day of lameness treatment plus $3 \mathrm{~d}$ before the treatment; lame $+5=$ day of lameness treatment plus $5 \mathrm{~d}$ before the treatment; lame $+7=$ day of lameness treatment plus $7 \mathrm{~d}$ before the treatment.

${ }^{2} \mathrm{k}=$ reference value constant, which is related to the magnitude of change to be detected; $\mathrm{h}=$ decision interval, which varied from a value of 0.1 to 18 .

${ }^{3}$ True positive.

${ }^{4}$ False positive.

${ }^{5}$ Average herd size: 78.2 cows per day.

${ }^{6}$ Average herd size: 67.1 cows per day.

with a SCC. Nevertheless, this approach did not lead to better results compared with this study. Classification results can also be influenced by the mastitis or lameness category itself. Due to low number of animals within the categories found or categories without further specification of, for example, mastitis, the categories were combined with cases of mastitis and of lameness, respectively.

According to Hogeveen et al. (2010), an alert should be given before clinical signs are visible so that treatment has greater efficiency. Therefore, disease blocks were analyzed before treatment occurred. Bareille et al. (2003) stated that mastitis affects milk production at $3 \mathrm{~d}$, whereas feed intake is disturbed by mastitis at around $4 \mathrm{~d}$ before the visual onset of this disease. Thus,
3- and 4-d periods before clinical signs were chosen for mastitis detection. Up to $5 \mathrm{~d}$ have been reported to identify lameness (e.g., Bareille et al. 2003). Furthermore, González et al. (2008) showed that lame cows change their feeding behavior in the $30-\mathrm{d}$ period before the disease occurs. Hence, 3-, 5-, and 7-d periods before clinical outbreak (i.e., an occurrence of the first treatment) were used for lameness detection.

However, the choice of the length of the disease blocks has widely varied ( 1 to $17 \mathrm{~d}$ ) in past research on disease detection (de Mol et al., 1997; Hogeveen et al., 2010; Kamphuis et al., 2010). For instance, de Mol and Woldt (2001) indicated $7 \mathrm{~d}$ before mastitis treatment occurred. Cavero et al. (2008) used disease blocks of 5 d (day of treatment plus $2 \mathrm{~d}$ prior and after treatment)

Table 6. Results of the multivariate vector autoregressive (VAR) models combined with self-starting multivariate cumulative sum (MCUSUM) charts requiring at least $70 \%$ block sensitivity

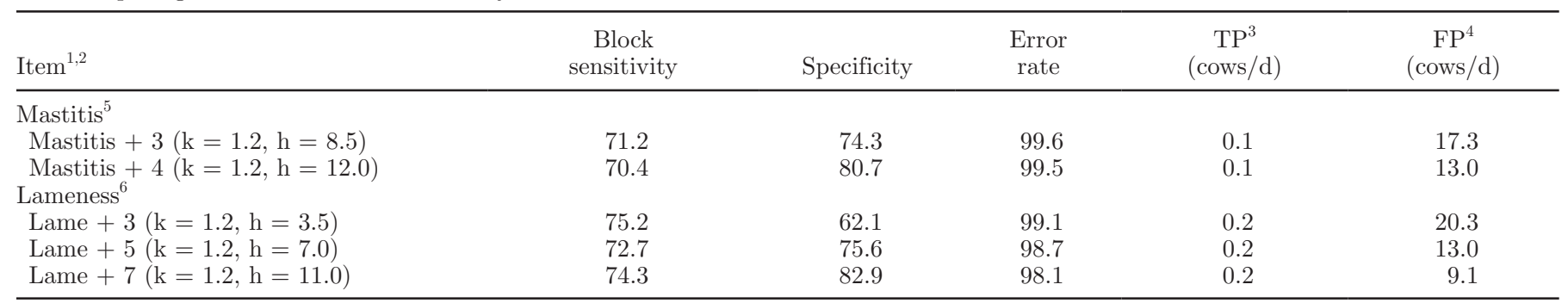

${ }^{1}$ Mastitis $+3=$ day of mastitis treatment plus $3 \mathrm{~d}$ before the treatment; mastitis $+4=$ day of mastitis treatment plus $4 \mathrm{~d}$ before the treatment; lame $+3=$ day of lameness treatment plus $3 \mathrm{~d}$ before the treatment; lame $+5=$ day of lameness treatment plus $5 \mathrm{~d}$ before the treatment; lame $+7=$ day of lameness treatment plus $7 \mathrm{~d}$ before the treatment.

${ }^{2} \mathrm{k}=$ reference value constant, which is related to the magnitude of change to be detected; $\mathrm{h}=$ decision interval, which varied from a value of 0.1 to 18 .

${ }^{3}$ True positive.

${ }^{4}$ False positive.

${ }^{5}$ Average herd size: 77.2 cows per day.

${ }^{6}$ Average herd size: 65.1 cows per day. 
for mastitis detection. In general, block sensitivity increases if longer periods are considered. In consequence, a comparison of model performance with other studies is difficult.

Sensitivity, specificity, accuracy, and error rate are well-known statistical quality parameters (Hogeveen et al., 2010). These parameters are used in several studies (e.g., Kramer et al., 2009; Lukas et al., 2009; De Vries and Reneau, 2010; Kamphuis et al., 2010) and in the International Organization for Standardization standard (ISO, 2007; ISO/FDIS 20966) to characterize the performance of a health detection method in dairy cows. Nonetheless, they are subjects of critical discussion (Hogeveen et al., 2010). As raw counts of mastitis or lameness events, they are independent of the occurrence of the event (mastitis or lameness) to be detected (Hogeveen et al., 2010), giving equal weight to each class (healthy and ill). The proportion between days of disease and days of health of mastitis as well as lameness is low, leading, for example, to high error rates. To account for this, some authors recommend the use of different quality parameters such as the success rate for evaluation, taking the prevalence of a disease into account (Sherlock et al., 2008; Hogeveen et al., 2010). A disadvantage of such an approach is that it is not an absolute statistic, varying due to seasonal and management changes and between farms. Therefore, in the current study, sensitivity, specificity, and error rate were used for characterizing the performance of the detection methods.

Miekley et al. (2012) analyzed a univariate mastitis as well as lameness monitoring system. They expected multivariate monitoring methods to possibly improve the results of disease detection systems, which is in concordance with Cavero et al. (2007). Comparing the findings of Miekley et al. (2012) with the current study show that for both diseases, especially lameness, sensitivity improved, whereas the other performance parameters differed slightly. The reason why the multivariate approach did not outperform the univariate monitoring system can be a result of the indicator variables. Due to their easy availability at reasonable costs, the sensormeasured data of MY and MEC have been used in several studies (Cavero et al., 2008; González et al., 2008; Hagnestam-Nielsen et al., 2009; Kramer et al., 2009; Lukas et al., 2009). Compared with the current study, Cavero et al. (2008), for example, achieved higher block sensitivities $(92.9 \%)$ and specificities $(93.9 \%)$ by application of neural networks. However, the MEC values of Cavero et al. (2008) were measured at quarter level of each cow, which allowed better detection performance (Hogeveen et al., 2010). Additionally, high variation exists of recorded traits between cows and also within cows, causing a high number of FP cows per day. The performance of the sensors currently used in practice are also criticized and should be improved considerably (Hogeveen et al., 2010). According to Mottram et al. (2002), the use of information from biosensors provides an opportunity to detect diseases, especially mastitis, more reliably than in currently used monitoring systems. Nevertheless, engineering progress and biological research is needed to integrate biosensors practically (Mottram et al., 2002).

Due to the fact that cows always react individually to diseases, several studies recommend individual cow analysis (Kramer et al., 2009; Lukas et al., 2009; Brandt et al., 2010). In general, all steps of each combined monitoring system used in this study enable such an approach.

Wavelet filters were able to adapt flexibly to the given input data without prior or additional information (e.g., stage of lactation) on each cow, supporting the idea of Kruse et al. (2011) and Miekley et al. (2012) on wavelets as an interesting tool for biological data preprocessing. However, missing data and necessary adjustments, such as the choice of the filter depth by the scientist, are some drawbacks for wavelets (Gençay et al., 2002; Miekley et al., 2012).

Other than univariate wavelet filters, multivariate VAR models take the relationships among several time series variables into account (Pfaff, 2008). However, multivariate preprocessing methods are not necessarily better than univariate approaches (Chatfield, 2001). Due to only marginally varying performance parameters between both preprocessing methods and due to the additional need for stationary data, this study comes to the same conclusion as that reached by Chatfield (2001).

Currently, no known scientific paper exists dealing with MCUSUM for the health management of livestock. Classic MCUSUM control charts, in general, are easy to implement and provide the herdsmen with one performance chart of each cow. For the user, however, it is not obvious which input variable from one chart, particularly at the time of an alert, might cause the most effect. Additionally, MCUSUM charts do not cope with missing data of one of the input variables, leading to omission of all of the existing information of the affected cow at this time (Waterhouse et al., 2010). This circumstance caused a loss of information up to $30 \%$ for some cows during their 200 DIM, influencing performance results in the current study.

The application of the self-starting transformation on the MCUSUM chart enables monitoring without prior information on each cow. To date, further development of MCUSUM charts, in particular in human health monitoring, is gaining attention (Waterhouse et al., 2010). For self-starting multivariate control charts, this 
progress in research is currently solely based on multivariate exponentially weighted moving average charts.

\section{CONCLUSIONS}

The automation of the detection of mastitis or lameness with MCUSUM charts using traits with regard to performance (MY, MEC, and feed intake) as well as behavior (feeding behavior and activity) revealed appealing features for individual disease detection in dairy cows. Multivariate extensions from the preprocessing method of wavelet filtering to the usage of the VAR model did not lead to further performance improvement. Without any changes to the settings of the study, high error rates as well as too many FP cows per day do not yet allow the practical implementation of one of the combined monitoring systems used. Possible enhancement of the performance of the monitoring systems might be achieved by the application of more reliable sensor data and different input traits.

\section{REFERENCES}

Athanasopoulos, G., and F. Vahid. 2008. VARMA versus VAR for macroeconomic forecasting. J. Bus. Econ. Stat. 26:237-252.

Bareille, N., F. Beaudeau, S. Billon, A. Robert, and P. Faverdin. 2003. Effects of health disorders on feed intake and milk production in dairy cows. Livest. Prod. Sci. 83:53-62.

Bodnar, O., and W. Schmid. 2007. Surveillance of the mean behavior of multivariate time series. Stat. Neerl. 61:383-406.

Brandt, M., A. Haeussermann, and E. Hartung. 2010. Invited review: Technical solutions for analysis of milk constituents and abnormal milk. J. Dairy Sci. 93:427-436.

Cavero, D., K.-H. Tölle, C. Henze, C. Buxadé, and J. Krieter. 2008. Mastitis detection in dairy cows by application of Neural Networks. Livest. Sci. 114:280-286.

Cavero, D., K. H. Tölle, G. Rave, C. Buxadé, and J. Krieter. 2007. Analysing serial data for mastitis detection by means of local regression. Livest. Sci. 110:101-110.

Chatfield, C. 2001. Time-Series Forecasting. Chapman \& Hall, London, UK.

da Cunha Alves, C., R. W. Samhohyl, and E. Henning. 2010. Application of multivariate cumulative sum charts (MCUSUM) for monitoring a machining process. XVI International conference on industrial engineering and operations management. ICIEOM, Sao Carlos, Brazil.

Daubechies, I. 1990. The wavelet transform, time-frequency localization and signal analysis. Information theory. IEEE Trans. Information Theory 36:961-1005.

de Mol, R. M., G. H. Kroeze, J. M. F. H. Achten, K. Maatje, and W. Rossing. 1997. Results of a multivariate approach to automated oestrus and mastitis detection. Livest. Prod. Sci. 48:219-227.

de Mol, R. M., and W. E. Woldt. 2001. Application of fuzzy logic in automated cow status monitoring. J. Dairy Sci. 84:400-410.

de Vries, A., and B. J. Conlin. 2003. Design and performance of statistical process control charts applied to estrous detection efficiency. J. Dairy Sci. 86:1970-1984.

De Vries, A., and J. K. Reneau. 2010. Application of statistical process control charts to monitor changes in animal production systems. J. Anim. Sci. 88(Suppl.):E11-E24.

Gençay, Z., F. Selçuk, and B. Whitcher. 2002. An Introduction to Wavelets and Other Filtering Methods in Finance and Economics. Academic Press, San Diego, CA.
González, L. A., B. J. Tolkamp, M. P. Coffey, A. Ferret, and I. Kyriazakis. 2008. Changes in feeding behavior as possible indicators for the automatic monitoring of health disorders in dairy cows. J. Dairy Sci. 91:1017-1028.

Hagnestam-Nielsen, C., U. Emanuelson, B. Berglund, and E. Strandberg. 2009. Relationship between somatic cell count and milk yield in different stages of lactation. J. Dairy Sci. 92:3124-3133.

Hawkins, D. M., and E. M. Maboudou-Tchao. 2007. Self-starting multivariate exponentially weighted moving average control charting. Technometrics 49:199-209.

Hawkins, D. M., and D. H. Olwell. 1998. Cumulative Sum Charts and Charting for Quality Improvement. Springer, New York, NY.

Hogeveen, H., C. Kamphuis, W. Steeneveld, and H. Mollenhorst. 2010. Sensors and clinical mastitis - The quest for the perfect alert. Sensors (Basel) 10:7991-8009.

Højsgaard, S., and N. C. Friggens. 2010. Quantifying degree of mastitis from common trends in a panel of indicators for mastitis in dairy cows. J. Dairy Sci. 93:582-592.

ISO (International Organization for Standardization). 2007. Automatic milking systems - Requirements and testing. Annex C: Example of methods of evaluating detection systems for milk deemed as abnormal due to blood or to changes in homogeneity. ISO 20966:2007. ISO, Geneva, Switzerland.

Kamphuis, C., H. Mollenhorst, A. Feelders, D. Pietersma, and H. Hogeveen. 2010. Decision-tree induction to detect clinical mastitis with automatic milking. Comput. Electron. Agric. 70:60-68.

Kramer, E., D. Cavero, E. Stamer, and J. Krieter. 2009. Mastitis and lameness detection in dairy cows by application of fuzzy logic. Livest. Sci. 125:92-96.

Kruse, S., I. Traulsen, J. Salau, and J. Krieter. 2011. A note on using wavelet analysis for disease detection in lactating sows. Comput. Electron. Agric. 77:105-109.

Lukas, J. M., J. K. Reneau, and J. G. Linn. 2008. Water intake and dry matter intake changes as a feeding management tool and indicator of health and estrus status in dairy cows. J. Dairy Sci. 91:3385-3394.

Lukas, J. M., J. K. Reneau, R. Wallace, D. Hawkins, and C. MunozZanzi. 2009. A novel method of analyzing daily milk production and electrical conductivity to predict disease onset. J. Dairy Sci. 92:5964-5976.

Lütkepohl, H. 1991. Introduction to Multiple Time Series Analysis. Springer Verlag, Berlin, Germany.

Lütkepohl, H. 2007. Econometric analysis with vector autoregressive models. Economics working Papers ECO2007/11. European University Institute, Florence, Italy.

Makridakis, S. G., S. C. Wheelwright, and R. J. Hyndman. 1988. Forecasting: Methods and Applications. John Wiley \& Sons, New York, NY.

MathWorks Inc. 2010. Wavelet Toolbox, for use with MATLAB ${ }^{\circledR}$ 7.10.0. The MathWorks Inc., Natick, MA.

Mertens, K., E. Decuypere, J. De Baerdemaeker, and B. De Ketelaere. 2011. Statistical control charts as a support tool for the management of livestock production. J. Agric. Sci. 149:369-384.

Miekley, B., I. Traulsen, and J. Krieter. 2012. Detection of mastitis and lameness in dairy cows using wavelet analysis. Livest. Sci. 148:227-236.

Milner, P., K. L. Page, and J. E. Hillerton. 1997. The effects of early antibiotic treatment following diagnosis of mastitis detected by a change in the electrical conductivity of milk. J. Dairy Sci. 80:859863.

Montgomery, D. C. 2009. Statistical Quality Control: A Modern Introduction. John Wiley and Son, Inc., Scottsdale, Arizona.

Mottram, T., M. Velasco-Garcia, P. Berry, P. Richards, J. Ghesquiere, and L. Masson. 2002. Automatic on-line analysis of milk constituents (urea, ketones, enzymes and hormones) using biosensors. Comp. Clin. Pathol. 11:50-58.

Patel, A., and J. Divecha. 2010. Modified cumulative sum quality control scheme. J. Eng. Technol. Res. 2:226-236.

Pfaff, B. 2008. Analysis of Integrated and Cointegrated Time Series with R. Springer Verlag, New York, NY. 
Pignatiello, J. J., Jr., and G. C. Runger. 1990. Comparisons of multivariate CUSUM charts. J. Quality Technol. 22:173-178.

SAS Institute. 2009. SAS/STAT User's guide. Release 9.1. SAS Institute Inc., Cary, NC.

Sherlock, R., H. Hogeveen, G. Mein, and M. Rasmussen. 2008. Performance evaluation of systems for automated monitoring of udder health: Analytical issues and guidelines. Pages 275-282 in Mastitis Control: From Science to Practice. Wageningen Academic Publishers, Wageningen, the Netherlands.
Waterhouse, M., I. Smith, H. Assareh, and K. Mengersen. 2010. Implementation of multivariate control charts in a clinical setting. Int. J. Qual. Health Care 22:408-414.

Wutsqa, D. U., Subanar, S. Guritno, and Z. Soejoeti. 2006. Forecasting performance of VAR-NN and VARMA models. Pages 194-200 in Proceedings of the 2nd IMT-GT Regional Conference on Mathematics, Statistics and Applications. Universiti Sains Malaysia, Pulau Penang, Malaysia. 